\title{
WIFI-based Smart Car for Toxic Gas Monitoring in Large-scale Petrochemical Plants
}

\author{
Lei Shu ${ }^{1}$, Junlin Zeng ${ }^{1}$, Kailiang $\mathrm{Li}^{1}$, Zhiqiang $\mathrm{Huo}^{2}$, Xiaoling $\mathrm{Wu}^{3}$, Huilin $\mathrm{Sun}^{1}$ \\ ${ }^{1}$ Guangdong Provincial Key Laboratory on Petrochemical Equipment Fault Diagnosis, \\ Guangdong University of Petrochemical Technology, Maoming, China \\ ${ }^{2}$ School of Information Engineering, China University of Geosciences, Beijing, China \\ ${ }^{3}$ Guangzhou Institute of Advanced Technology, Chinese Academy of Sciences \\ 1121 Haibin Rd. Nansha District, Guangzhou, China \\ Email: lei.shu@ieee.org,junlin.zeng@outlook.com, kailiang.li@outlook.com, zhiqiang.huo@outlook.com, \\ xl.wu@giat.ac.cn, huilin.sun@outlook.com
}

\begin{abstract}
Providing complete monitoring on the concentration of various toxic gases in large-scale petrochemical plants is critical, since it serious affects the safely producing activities and first line workers' lives. Safe production environment can enhance the productivity and keep high profits of enterprises. In this paper, we present a newly developed mobile car with WIFI wireless communication to smartly monitor and track the concentration of various toxic gases.

Index Terms-Gas monitoring; Smart car;
\end{abstract}

\section{The Research Problem}

Safety is a problem which annually receives significant consideration and investment in oil and gas industries, e.g., large-scale petrochemical plants. In industrial plants, producing equipment may be corroded due to long-term contacting with caustic chemical materials. This kind of corrosion can easily lead to serious disasters as toxic and combustible gas leakage can severely damage the workers health and profits of enterprises. For examples, in recent years, there are a few very serious incidents happened in major petrochemical plants in China: 1) toxic gas leakage in Haidong of Qinghai province in 2014,2 ) toxic gas leakage in Baoshan of Shanghai city in 2014, 3) fire incident in Changyang of Hubei province in 2012. All of these incidents caused a large number of lives lost and long time environment pollution.

At present, to the best of our knowledge, in most major petrochemical plants in China, the existing toxic gases concentration monitoring system consists of two parts: 1) static cable connected sensor nodes; 2) hand-held sensing devices of first line workers. When toxic gas leakage is detected by static cable connected sensor nodes, these workers will go to the corresponding area to check the leaking point. Normally, these workers will judge the toxic gas leaking source based on the direction of concentration change, wind direction, and professional experience. However, this kind of monitoring mechanism is limited by finite workers' working time and specific ares. We can see that there are several obvious disadvantages in traditional monitoring mechanism:

- Low efficiency of monitoring work, workers with enough real working experience are enable to make right forecasts and judges.

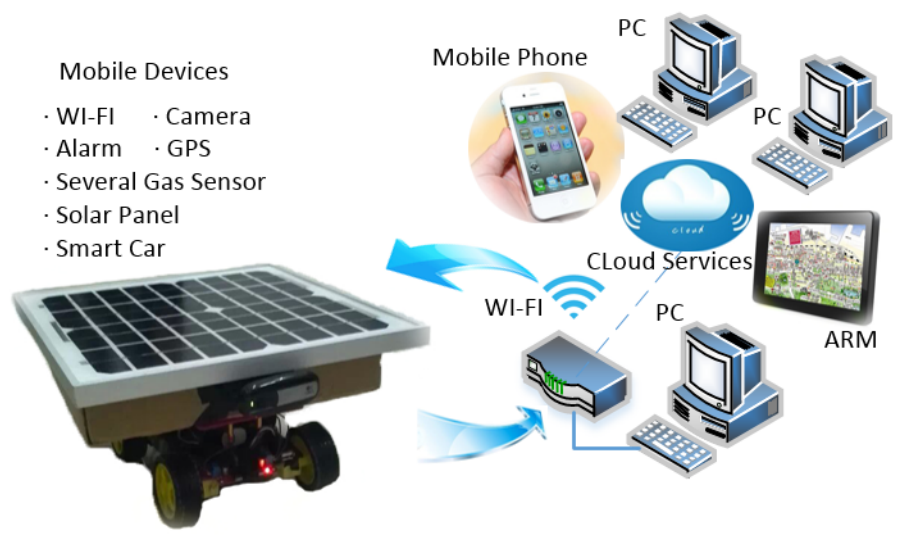

Fig. 1: In this figure, smart monitoring car on the left is shown as one type of mobile sensing nodes, which is developed by IWSNLab. This smart car is equipped with various sensors, e.g., $\mathrm{CO}, \mathrm{SO}_{2}, \mathrm{H}_{2} \mathrm{~S}$, humidity, video and audio sensors. It is connected with control platform and remote server for controlling and comprehensive on-line monitoring in petrochemical plants.

- Limited monitoring areas, some regions in industrial plants are remote and hard-to-reach even prohibited access of workers.

- Sensing data can not be stored, processed, analyzed, shared and used for knowledge discovery.

- Health of workers may be threatened when measuring in potential dangerous region.

\section{Solutions}

The instinct of mobility allows observing unpredictable events, e.g., toxic gas leakage, which cannot be detected by static sensor nodes in finite areas. In cooperation of mobile and static mechanisms provides more large spatial coverage. To solve these issues above mentioned in traditional measuring method, we propose a basically intelligent real-time mechanism. For real-time toxic gas monitoring in industrial environment, we can apply mobile gas sensing nodes to cooperate with static sensor nodes. Those mobile sensing nodes are able 
to be realized in various methods, e.g., mobile cars, mobile robots and even workers with wearable and portable sensing devices which is known as "workers as sensors".

Combination of Internet of Things (IoT) and cloud computing techniques, this type of various gas data can contribute to large-scale gas monitoring, analysis, on-line visualization and timely emergency reporting. This is not only vitally important to understand nature (e.g., correlations and contexts) of the data for knowledge discovery, but also make workers really participate in high-efficiency systems without the limit of professional knowledge. With real-time monitoring and design-making, this intelligent pattern facilitates less lost and increased safety for keeping high profits of enterprises.

\section{SMART Monitoring CAR}

Based on the innovated monitoring patterns and our existing on-line gas monitoring in oil and gas industry. We developed WIFI-based smart monitoring car with mobility and intelligence. This smart car is easy-implement,easy-operate, lowcost and flexible to move on the ground. With WIFI technique, the mobile monitoring car owns the ability of transmitting large types of sensing data, e.g., video and audio data.

We apply industrial WIFI router for making communication between smart car and terminal server. The range radius of signal covers one hundred meter. This type of WIFI technique supports UDP and TCP/IP Internet protocol, 802.11/b/g/n wireless standards and UART/GPIO Ethernet communication interfaces.

At the front of mobile monitoring car, there is five million pixels of the camera. Workers use this camera for navigation and capturing real-time video data. With the GPS module, the visualization of moving traces is presented on the control equipment and terminal server.

This type of car is equipped with various gas sensors, e.g., $\mathrm{CO}_{2}, \mathrm{CO}, \mathrm{SO}_{2}, \mathrm{H}_{2} \mathrm{~S}$. Among those sensors, the type of $\mathrm{CO}_{2}$ sensor with NDOR is $\mathrm{K} 30$. Its measuring range is 0 to 5000 $\mathrm{ppm}$, and precision is $30 \mathrm{ppm}$. The others are electrochemical sensors whose measuring range is 0 to $5000 \mathrm{ppm}$ and precision is $0.1 \mathrm{ppm}$. In addition, this car is also equipped with other types of sensors, e.g., humidity and temperature sensors. All the environment sensing data can contribute to comprehensive environment data gathering and analysis so that leading to increased safety and less logistic cost.

Smart monitoring car is supported by solar power and battery. It will be charged when the light source available. When the energy is not enough, it will send messages to control center and turn itself into sleep status. At this time, smart car will be periodically waked up in order to detect light source for charging.

\section{IMPACTS FOR SCIENTIFIC STUDY}

Safe production environment can enhance the productivity of petrochemical factories, which is commonsense. Scientists had already tried to monitor toxic gas leakage and noise pollution for further study. Research work in this paper reveals two important insights as follows:

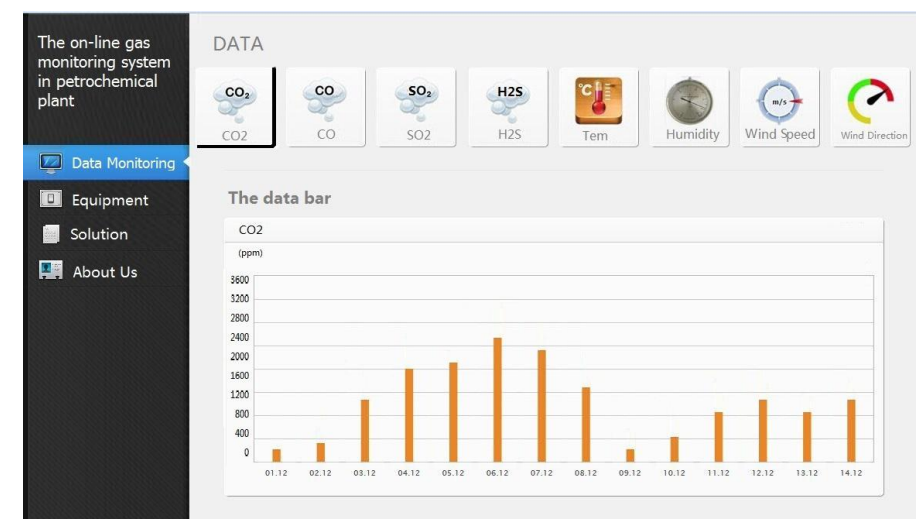

Fig. 2: In this figure, on-line gas monitoring system in petrochemical plant is shown. Presentation of real-time sensing data is on the terminal monitoring system, e.g., the concentration of toxic gas, humidity and wind speed in surrounding environment. Bar chart below shows the changes of $\mathrm{CO}_{2}$ gas data by date.

- The credibility of data gathered by workers need to be evaluated. Environment data gathered from various sensing devices through workers may be polluted due to some reasons, e.g., different intentions, different sensing operations and activities. Necessary context-aware, localization, data processing functions can enhance the quality and credibility of this type of data. It is one challenge to design adaptive strategies to assess and process these types of data to make it useful.

- Real-time gas monitoring map is critical to take actions for preventing emergency events, e.g., toxic gas leakage. Different from noise map, the distribution of toxic gas map is dynamic extremely. It is challenge to timely evaluate the status of incidents and find the leakage sources with professional domain knowledge. Thus, how to realize toxic gas map visualization and conduct decision-making processes in real-time need a further study.

\section{ACKNOWLEDGMENT}

This work is supported by 2013 Special Fund of Guangdong Higher School Talent Recruitment, Educational Commission of Guangdong Province, China Project No. 2013KJCX0131, Guangdong High-Tech Development Fund No. 2013B010401035, 2013 top Level Talents Project in "Sailing Plan" of Guangdong Province, National Natural Science Foundation of China (Grant NO. 61401107), and 2014 Guangdong Province Outstanding Young Professor Project.

\section{REFERENCES}

[1] K. Wang,H. Lu,L. Shu and Joel J.P.C.Rodrigues, "A Context-aware System Architecture for Leak Point Detection in Large-scale Petrochemical Industries. In IEEE Communications Magazine, 2014.

[2] R. Rana,C .T.Chou,S .Kanhere,N. Bulusu and W. Hu, "Ear-Phone:An End-to-End Participatory Urban Noise Mapping System”. In Processdings of ACM/IEEE IPSN, Stockholm, Sweden, 2010. 